\title{
BioéthiqueOnline
}

\section{La conciliation travail-famille : le défi des mères médecins}

\section{Claude Johnson et Vardit Ravitsky}

Volume 4, 2015

Reçu : 11 Apr 2015; publié : 3 Jun 2015; éditeurs : Vincent Couture \& Hazar Haidar; éditeur de section par intérim : Charles Marsan

URI : https://id.erudit.org/iderudit/1035499ar

DOI : https://doi.org/10.7202/1035499ar

Aller au sommaire du numéro

Éditeur(s)

BioéthiqueOnline

ISSN

1923-2799 (numérique)

Découvrir la revue

Citer cet article

Johnson, C. \& Ravitsky, V. (2015). La conciliation travail-famille : le défi des mères médecins. BioéthiqueOnline, 4. https://doi.org/10.7202/1035499ar

\section{Résumé de l'article}

Les médecins, compte tenu des exigences de leur profession, présentent d'importantes difficultés de conciliation travail-famille. Des mesures favorisant la conciliation travail-famille seraient bénéfiques pour tous les médecins (particulièrement pour les femmes), leurs enfants et leurs patients.
Droits d'auteur (C) C Johnson et V Ravitsky, 2015

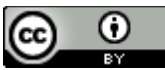

Ce document est protégé par la loi sur le droit d'auteur. L'utilisation des services d'Érudit (y compris la reproduction) est assujettie à sa politique d'utilisation que vous pouvez consulter en ligne.

https://apropos.erudit.org/fr/usagers/politique-dutilisation/ 


\title{
La conciliation travail-famille : le défi des mères médecins
}

\author{
COMMENTAIRE / COMMENTARY \\ Claude Johnson ${ }^{1}$, Vardit Ravitsky ${ }^{1}$
}

Reçu/Received: 11 Apr 2015

Publié/Published: 3 Jun 2015

Éditeurs/Editors: Vincent Couture \& Hazar Haidar

Éditeur de section par intérim/Interim Section Editor: Charles Marsan

2015 C Johnson, V Ravitsky, Creative Commons Attribution 4.0 International License

\section{Résumé}

Les médecins, compte tenu des exigences de leur profession, présentent d'importantes difficultés de conciliation travail-famille. Des mesures favorisant la conciliation travail-famille seraient bénéfiques pour tous les médecins (particulièrement pour les femmes), leurs enfants et leurs patients.

\section{Mots clés}

conciliation travail-famille, conflits travail-famille, projet de loi no 20 , médecins, féminisation, profession médicale, éthique, enfants

\section{Summary}

Doctors, given the requirements of their profession, have important work-family balance challenges. Measures supporting work-life balance would be beneficial for all physicians (especially for women), their children and their patients.

\section{Keywords}

work-life balance, work-family conflict, Bill 20, doctors, feminization, medical profession, ethics, children

Affiliations des auteurs / Author Affiliations

${ }^{1}$ Programmes de bioéthique, Département de médecine sociale et préventive, École de santé publique de l'Université de Montréal, Montréal (Québec), Canada

\section{Correspondance / Correspondence}

Claude Johnson, claude.johnson@umontreal.ca

\section{Remerciements}

Les auteures tiennent à remercier tous les médecins qui, par leur engagement envers la profession médicale et auprès de leurs enfants, contribuent à améliorer la société d'aujourd'hui et à préparer celle de demain.

\section{Conflit d'intérêts}

Hazar Haidar, éditrice à BioéthiqueOnline, est étudiante au doctorat sous la supervision de Professeure V. Ravitsky.

\section{Acknowledgements}

The authors would like to thank all the doctors who, through their commitment to the medical profession and to their children, help to improve today's society and prepare for that of tomorrow.

\section{Conflicts of Interest}

Hazar Haidar, editor at BioéthiqueOnline, is a PhD candidate under the supervision of Professor V. Ravitsky.

\section{Introduction}

Le Québec vit actuellement une crise sans précédent au niveau de la réorganisation des soins de santé. Les transformations dramatiques du système de soins et de la société, au cours des dernières décennies, ont fait en sorte qu'un énorme fossé s'est créé entre les services offerts par la profession médicale et les attentes de la population [1,2]. Ces attentes créent un fardeau pour les médecins, qui augmente les tensions qu'ils vivent par rapport à la conciliation travail-famille. Ces tensions posent un défi particulier pour les femmes médecins, qui, malgré le progrès important dans le domaine de l'équité homme-femme, se trouvent souvent avec plus de responsabilités domestiques que leurs collègues masculins.

La profession médicale impliquant de grandes responsabilités, les mères-médecins se sentent souvent déchirées entre leur rôle de mère et celui de médecin. D'un côté, leur responsabilité sociale exige qu'elles soient au service des patients et de l'autre, leur responsabilité parentale demande qu'elles soient présentes pour les soins et l'éducation de leurs enfants. Ce conflit représente un enjeu 
d'actualité en lien avec le débat autour de la réorganisation des soins de santé. Visant à alimenter la réflexion concernant les enjeux liés au fait d'être mère et médecin, ce commentaire s'articule dans l'effervescence entourant la controverse suscitée par le dépôt du projet de loi 20 par le ministre de la Santé et Services sociaux, Dr. Gaetan Barette, en novembre 2014. II met en lumière des tensions entre les responsabilités médicales et parentales et offre un argument en faveur de mesures favorisant la conciliation travail-famille pour les médecins, plus particulièrement pour les femmes, en raison de leur réalité actuelle dans la société.

De telles mesures favoriseront non seulement une meilleure qualité de vie pour les femmes médecins, mais auront aussi un impact positif sur la qualité des soins offerts à leurs patients et sur le développement et la santé de leurs enfants. Le projet de loi 20 , en suggérant que les médecins ne travaillent pas assez et qu'ils devraient en faire plus, irait dans une direction opposée à celle offerte ici, ce qui pourrait amener des conséquences néfastes au point de vue individuel pour les femmes médecins et leurs enfants, et au niveau sociétal pour leurs patients et l'ensemble du système de santé.

\section{Être médecin : une responsabilité sociale}

La médecine est une profession qui implique de grandes responsabilités en raison des particularités reliées à la santé [3]. La santé est considérée par plusieurs comme étant un bien "spécial ", qui doit être traité différemment des autres biens sociaux [3]. La santé n'est pas considérée au même niveau que les autres produits de consommation, mais considérée comme faisant partie des biens publics d'une société et aussi comme un droit [3-7]. Certaines caractéristiques aident à comprendre pourquoi la santé et les soins requis pour remédier à la maladie font en sorte que la problématique de l'accès aux soins est spéciale et difficile à résoudre.

D'une manière générale, les soins de santé créent toujours des dilemmes moraux en terme de justice, parce que les besoins sont nombreux et dépassent inévitablement les possibilités d'y répondre [8], et avec les avancées de la médecine, les gammes de traitements se multiplient. Les conséquences de ne pas répondre aux besoins en matière de soins de santé sont particulières : elles risquent de mettre la vie en péril, porter atteinte à l'intégrité physique des patients ou menacer leur intégrité psychologique pour ce qui est du domaine de la santé mentale. Bien qu'on reconnaisse que les médecins du Québec offrent des soins de qualité à leurs patients, on leur reproche leur manque de responsabilisation en matière d'offre de services à la population $[9,10]$. Bien que plusieurs politiciens et citoyens considèrent que les médecins ne travaillent pas assez, plusieurs d'entre eux trouvent que leurs horaires, conditions de travail et responsabilités sont stressants [11]. II existe donc un décalage entre les attentes de la société et la capacité de travail des médecins.

Une étude canadienne rapporte que $64 \%$ des médecins considèrent que leur charge de travail est trop lourde, et qu'ils travaillent environ 50-60 heures par semaine en plus des gardes [12,13]. Selon une grande étude américaine récente, $37.9 \%$ des médecins souffrent d'épuisement professionnel alors que le taux d'épuisement professionnel pour une population comparable (âge, sexe, statut social, niveau d'éducation, etc.) est de $27.8 \%$. De plus, ils travaillent en moyenne 10 heures de plus par semaine que le groupe contrôle [14]. D'un côté, la population et les politiciens demandent aux médecins d'en faire plus, et de l'autre, les médecins trouvent qu'ils en font trop et se sentent épuisés. «La perception qu'a la société des médecins, soit le fait qu'ils sont des privilégiés, hautement éduqués, bien rémunérés pour un travail qui est reconnu et valorisé n'est pas conforme à la réalité » [15], et leurs difficultés sont bien documentées dans la littérature [16-18]. La perception sociale que les médecins ne prennent pas leurs responsabilités serait en fait fausse, c'est plutôt qu'ils sont incapables de répondre à la demande en raison d'un épuisement. Les statistiques concernant l'épuisement professionnel impliquent que l'origine du problème se situe dans l'environnement de travail (conditions et organisation du travail), plutôt que secondaire à une faiblesse de quelques individus [14]. 


\section{Féminisation de la profession médicale}

Durant des siècles, la profession médicale était réservée aux hommes, mais le mouvement féministe a grandement contribué à la féminisation de la profession. L'arrivée massive des femmes en médecine a eu des impacts sur la façon d'exercer la médecine. Des études démontrent que les femmes exercent la médecine différemment des hommes. Elles travaillent moins d'heures que leurs collègues masculins (49 vs 56 heures par semaines) [19], mais passent plus de temps avec leurs patients, s'occupent de plusieurs problèmes différents au cours d'une visite, prescrivent moins de médicaments et sont plus axées sur la prévention [20,21]. Leur utilisation optimale des ressources, leur approche holistique orientée vers la prévention et leur implication auprès des clientèles vulnérables peuvent contribuer à améliorer l'accès aux soins et la qualité des services à long terme [22]. En général, les femmes ont aussi plus d'empathie envers leurs patients, ce qui est un point positif pour la qualité des soins [23].

La féminisation de la profession médicale a aussi mis en évidence la problématique de la conciliation travail-famille. Dans un monde idéal, les deux parents se partageraient les responsabilités familiales d'une manière équitable, en fonction de leurs besoins et préférences au cours des années, et ce, au bénéfice de toute la famille. En réalité, même à nos jours, la recherche démontre que les femmes en font plus que leurs conjoints pour les soins et l'éducation des enfants, et ce malgré des exigences professionnelles très élevées [24]. En conséquence, même dans la société québécoise actuelle qui valorise l'égalité homme-femme, les femmes se trouvent dans des situations où elles vivent des tensions importantes.

\section{La difficile conciliation travail-famille}

Une étude nationale effectuée aux États-Unis auprès de 7288 médecins a démontré que $40.2 \%$ des médecins sont insatisfaits de l'équilibre travail-vie personnelle, tandis que l'insatisfaction est de l'ordre de $23.2 \%$ pour une population comparable (en désaccord ou fortement en désaccord avec la question: "Mon horaire de travail me laisse suffisamment de temps pour ma vie personnelle/familiale ») [14]. Les difficultés de conciliation travail-famille chez les médecins peuvent être une source de stress et d'épuisement professionnel ce dont, rappelons-le, ils souffrent plus que d'autres professionnels (37.9 \% vs $27.8 \%$ ) [14,17,25,26].

Une corrélation existe entre les conflits travail-famille, la santé mentale du parent, l'irritabilité parentale et les difficultés des enfants. Les conflits travail-famille ont alors des conséquences néfastes sur la santé mentale des enfants et des adolescents (détresse émotionnelle, problèmes de comportement, difficultés relationnelles) [27]. Puisque la qualité de la relation parent-enfant est le facteur déterminant dans le développement psychosocial et cognitif des enfants et des adolescents, le statut socio-économique élevé des médecins ne compense pas pour les tensions relationnelles et leurs conséquences préjudiciables sur les enfants [28-30].

\section{Être mère et médecin}

Les modèles de rôle sont d'une importance primordiale pour les médecins en formation et ils font souvent exclusion de la parentalité [31]. Pour les femmes en médecine, la construction de l'identité professionnelle laisse très peu de place à la création d'une identité personnelle de mère. L'isolement, les modèles de rôle déficients et les exigences de la profession ne favorisent pas l'accomplissement à la fois professionnel et personnel.

Or, dans le cas des femmes médecins, les progrès importants au niveau de leur autonomie leur permettant d'intégrer le marché du travail, les obligent à payer un prix en tant que mères. Les femmes 
ont parfois l'impression de devoir sacrifier une partie d'elles-mêmes. Les femmes médecins se retrouvent donc dans une situation particulièrement difficile, déchirées entre des messages sociaux contradictoires. D'un côté, leur identité professionnelle de médecin les incite à se dévouer entièrement à leur vie professionnelle afin de remplir leurs obligations envers leurs patients et la société. D'un autre côté, on s'attend à ce qu'elles remplissent leurs obligations maternelles et prennent même plus de responsabilités que leurs conjoints. Elles croient vivre dans une société égalitaire leur permettant d'avoir plus de choix que par le passé, mais en fait, elles doivent faire d'énormes sacrifices. Soit qu'elles compromettent leur capacité à exercer la médecine telle qu'on leur a enseigné, soit qu'elles compromettent les soins et l'éducation qu'elles souhaitent offrir à leurs enfants.

\section{Peut-on concilier la maternité, des enfants en santé avec la carrière?}

En 2011, le ministère de la Famille du Québec lançait la norme conciliation travail-famille, énonçant les exigences en matière de bonnes pratiques dans ce domaine [32]. Ensuite, à l'initiative de la Commission canadienne de la santé mentale, des normes gouvernementales en santé et sécurité psychologique au travail ont été élaborées et publiées en 2013. Treize facteurs ont été retenus, dont " l'équilibre » et " la gestion de la charge de travail » [33]. Le but de cet ouvrage est de servir d'outil de référence afin de permettre l'instauration de ces normes et de faire la promotion de la santé psychologique en milieu de travail. II y a donc une volonté politique d'améliorer la santé des travailleurs. On reconnaît qu'un travailleur en santé est plus productif.

En 2014, le Collège royal des médecins et chirurgiens du Canada envoyait à tous ses membres un communiqué intitulé "Le Canada doit investir davantage dans le développement de la petite enfance » [34]. Le Collège a formulé plusieurs recommandations afin de proposer des mesures aux gouvernements et aux médecins pour améliorer la santé et le bien-être des enfants en bas âge, dont le soutien aux parents [35].

$\mathrm{Si}$, d'une manière cohérente, on intègre les connaissances scientifiques dans le domaine de la santé psychologique au travail, de la conciliation travail-famille et du développement du cerveau humain, on réalise l'importance d'avoir des politiques gouvernementales soutenant la conciliation travail-famille pour tous les parents. Des mesures soutenant les médecins devraient aussi être une priorité, compte tenu des difficultés élevées à concilier le travail et la famille dans cette profession. Avec une volonté politique et sociale, il est tout à fait possible de concilier la maternité, des enfants en santé et la carrière, même pour les femmes médecins. Concilier le travail et la famille pour les médecins, dans le contexte actuel du manque d'accès aux soins, ne veut pas nécessairement dire en faire moins pour la société et plus pour la famille du médecin. Cela veut dire trouver des solutions créatives afin de mieux respecter les besoins de toutes les parties prenantes. La santé d'une population ne se mesure pas à partir de la productivité de ses médecins, évaluée à partir d'un certain nombre d'actes médicaux. Investir dans les traitements en négligeant la prévention risque de conduire la société à des dérives éthiques et à une inefficience à long terme [36]. Les déterminants sociaux de la santé tels que les habitudes de vie, l'environnement de travail, les facteurs psychosociaux et le milieu de vie ont un très grand rôle à jouer dans la santé d'une population [37]. Une réorganisation du système de soins favorisant la collaboration interprofessionnelle pourrait aider à atteindre l'objectif de concilier le travail et la famille pour les médecins [38].

\section{Conclusion}

Le défi des femmes médecins de devoir répondre aux besoins de la société en matière de soins et en même temps de répondre aux besoins de leurs enfants place ces médecins dans une position difficile. Les exigences particulières reliées à leur travail et leur rôle traditionnel dans l'éducation des enfants sont trop souvent une source de tensions pouvant être à l'origine d'une détresse 
psychologique. L'impact de cette détresse sur les enfants est majeur en raison des effets sur la qualité relationnelle, si importante dans le développement des enfants et des adolescents.

Des mesures facilitant la conciliation travail-famille pour les médecins, et plus particulièrement pour les femmes médecins, seraient bénéfiques non seulement pour leur propre santé, mais aussi pour la santé de leurs patients, de leurs enfants et de la société en général. Paradoxalement, des normes permettant d'améliorer la santé et la sécurité psychologique sont reconnues et acceptées par les gouvernements et les organisations médicales, mais elles semblent s'adresser à tous sauf aux médecins.

Tandis que les politiques de santé visent généralement à favoriser le soutien aux parents, elles visent à mettre plus de pression sur les médecins en créant des lois pour les faire travailler davantage $[39,40]$. Les enfants de parents-médecins ne devraient pas subir les impacts négatifs sur leur développement psychosocial et cognitif de la difficile conciliation travail-famille de leurs parentsmédecins. Comme tous les enfants, ils ont le droit, eux aussi, d'avoir des parents présents et attentionnés. Un meilleur partage des responsabilités est souhaitable en ce qui concerne à la fois les soins de santé et la charge des enfants.

\section{Références}

1. AMQ. La profession médicale: vers un nouveau contrat social, Document de réflexion préparé par l'Association médicale du Québec, février 2015.

2. Cohen JJ, Cruess S, Davidson C. Alliance between society and medicine: the public's stake in medical professionalism. JAMA. 2007 Aug 8;298(6):670-3.

3. Daniels N. Health-Care Needs and Distributive Justice. Philosophy and Public Affairs. 1981;10(2):146-79.

4. Beauchamp TLF, Faden R. The Right to Health and the Right to Health Care. The Journal of Medecine and Philosophy. 1979;4(2):118-31.

5. Buchanan AE. The Right to a Decent Minimum Health Care. Philosophy \& Public Affairs. 1984;13(1):55-78.

6. Daniels N. Justice, Health, and Healthcare. The American Journal of Bioethics. 2001;1(2):2-16.

7. Daniels N. Just Health: Meeting Health Needs Fairly, New York: Cambridge University Press; 2008.

8. Tronto J. Un monde vulnérable: pour une politique du care. Paris: La Découverte; 2009.

9. Duquette P. La pression sur les médecins. LeDroit. 13 décembre 2014.

10. Belley C. Les omnipraticiens travaillent-ils assez? ICI Radio Canada. 19 décembre 2014

11. Wallace JE, Lemaire JB, Ghali WA. Physician wellness: a missing quality indicator. Lancet. 2009 Nov 14;374(9702):1714-21.

12. CMA. Canadian Medical Association. Guide to physician health and well-being: facts, advice and resources for Canadian doctors. Ottawa, Ontario: Canadian Medical Association; 2003.

13. Williams ES, Rondeau KV, Xiao Q, Francescutti LH. Heavy physician workloads: impact on physician attitudes and outcomes. Health services management research : an official journal of the Association of University Programs in Health Administration / HSMC, AUPHA. 2007 Nov;20(4):261-9.

14. Shanafelt TD, Boone S, Tan L, Dyrbye LN, Sotile W, Satele D, et coll. Burnout and satisfaction with work-life balance among US physicians relative to the general US population. Archives of Internal Medicine. 2012 Oct 8;172(18):1377-85.

15. Descary P. Rapport spécial de l'ombudsman, Soutien aux résidents en difficulté: constats et recommandations, Université de Montréal, mars 2015.

16. Adam S. [High prevalence of work-family conflict among female physicians: lack of social support as a potential antecedent]. Orvosi hetilap. 2009 Dec 13;150(50):2274-81.

17. Adam S, Gyorffy Z, Laszlo K. [High prevalence of job dissatisfaction among female physicians: work-family conflict as a potential stressor]. Orvosi hetilap. 2009 Aug 2;150(31):1451-6. 
18. Estryn-Behar M, Fry C, Guetarni K, Aune I, Machet G, Doppia MA, et al. Work week duration, work-family balance and difficulties encountered by female and male physicians: results from the French SESMAT study. Work. 2011;40 Suppl 1:S83-100.

19. Martin S. Family matters. CMAJ. 2003;168(9):1174.

20. Hedden L, Barer ML, Cardiff K, McGrail KM, Law MR, Bourgeault IL. The implications of the feminization of the primary care physician workforce on service supply: a systematic review. Human Resources for Health. 2014;12:32.

21. Levinson W, Lurie N. When most doctors are women: what lies ahead? Annals of Internal Medicine. 2004 Sep 21;141(6):471-4.

22. Contandriopoulos A, Fournier MA. Féminisation de la profession médicale et transformation de la pratique au Québec. Étude réalisée à la demande de l'Association Médicale du Québec: Université de Montréal; novembre 2007.

23. Hojat M, Gonnella JS, Mangione S, Nasca TJ, Veloski JJ, Erdmann JB, et al. Empathy in medical students as related to academic performance, clinical competence and gender. Medical Education. 2002 Jun;36(6):522-7.

24. Tremblay D-G. Conciliation emploi-famille et temps sociaux. Presses de l'Université du Québec; 2012.

25. Dyrbye LN, Shanafelt TD, Balch CM, Satele D, Sloan J, Freischlag J. Relationship between work-home conflicts and burnout among American surgeons: a comparison by sex. Archives of Surgery. 2011 Feb;146(2):211-7.

26. Adam S, Gyorffy Z, Susanszky E. Physician burnout in Hungary: a potential role for work-family conflict. Journal of Health Psychology. 2008 Oct;13(7):847-56.

27. Strazdins L, Obrien LV, Lucas N, Rodgers B. Combining work and family: rewards or risks for children's mental health? Social Science \& Medicine. 2013 Jun;87:99-107.

28. Siegel DJ. Attachment. The Developing Mind: How Relationships and the Brain Interact to Shape Who We Are. New York, London: Guilford Press; 1999. p. 67-120.

29. Siegel D. Toward an interpersonal neurobiology of the developing mind: Attachment relationships, 'mindsight', and neural integration. Infant Mental Health Journal. 2001;22(1-2):6794.

30. Cassidy J, \& Shaver, P.R. (Eds). Handbook of Attachment. New York: Guilford Press; 1999.

31. Ferris LE, Mackinnon SE, Mizgala CL, McNeill I. Do Canadian female surgeons feel discriminated against as women? CMAJ. 1996 Jan 1;154(1):21-7.

32. BNQ. La norme Conciliation travail-famille (BNQ 9700-820), Bureau de normalisation du Québec. 2011.

33. BNQ. Santé et sécurité psychologiques en milieu de travail- Prévention, promotion et lignes directrices pour une mise en oeuvre par étapes (CSA-Z1003-13/BNQ 9700-803) Bureau de normalisation du Québec/ Conseil canadien des normes/ Groupe CSA, élaborée à l'initiative de la Commission canadienne de la santé mentale. 2013.

34. Andrew Padmos M, FRCPC, FACP. Le Canada doit investir davantage dans le développement de la petite enfance. CRMCC. 2014.

35. CRMCC. Énoncé de position du Collège royal: Développement de la petite enfance. 2014.

36. Bergeron N. Les dérives éthiques du projet de loi 20: L'association entre l'offre quantitative de services médicaux et la santé de la population est réductrice et dangereuse. Le Devoir. 9 mai 2015.

37. St-Arnaud-Trempe E, Defay F, Markon M-P, Springmann V. État de situation sur la santé des Montréalais et ses déterminants. 2014.

38. Borgès Da Silva R, Sicotte C, Pineault R, Lauzon A-J, Blais R, Hébert R, Leduc N, Ouimet M-J.. Mémoire sur la première partie du projet de loi 20: loi favorisant l'accès aux services de médecine de famille et de médecine spécialisée, Mémoire rédigé par un regroupement de chercheurs en organisation des services de santé de l'Université de Montréal, mars 2015.

39. Auger S. Projet de loi 20: salaires réduits pour les médecins peu assidus. Le Soleil. 29 novembre 2014. 
40. Robitaille A. Indispensable Bâton: Projet de loi 20 sur l'accès aux soins. Le Devoir. 29 novembre 2014. 